\title{
Linear Equilibrium Adsorbing Solute Transport in Physically and Chemically Heterogeneous Porous Formations 2. Numerical Results
}

\author{
Willem Jan P. Bosma, ${ }^{1}$ Alberto Bellin, ${ }^{2,3}$ Sjoerd E. A. T. M. van Der Zee, 1 \\ AND ANDREA RINALDO 4
}

\begin{abstract}
Numerical Monte Carlo simulations were conducted to assess dispersion of reactive solutes in two-dimensional physically and chemically heterogeneous porous media, using random fields with assigned correlation structure for hydraulic conductivity and linear adsorption coefficient. Conditions under which linearization of adsorption is valid are discussed. Lognormal distributions of hydraulic conductivity and adsorption coefficient were assumed. Calculations have been performed for positive and negative correlation between hydraulic conductivity and adsorption coefficient, and for uncorrelated cases. Effects of varying different properties including mean and average sorption coefficient, physical and chemical integral scale, and variance of hydraulic conductivity on dispersive behavior are shown. A larger mean sorption coefficient enhances plume spreading in uncorrelated and in negatively correlated cases. In positively correlated cases, counteracting effects of physical and chemical heterogeneity play an important role. The outcome of these counteracting effects depends on the mean, variance, and integral scales of the spatially variable properties. The analytical solutions, derived in paper 1 (Bellin et al., this issue), reveal a good agreement with the numerical results in a significant range of heterogeneities. The generally surprisingly good agreement of the analytical solutions with the numerically obtained results can possibly be attributed to opposing effects of nonlinearities neglected in the derivation of the analytical solutions. In the case of strong physical heterogeneity the analytical solutions perform slightly better than in the case of strong chemical heterogeneity.
\end{abstract}

\section{INTRODUCTION}

From an environmental point of view, solute transport and its implications are an important area of research. In recent years, much attention has been addressed toward characterization of spatial variability of field-scale properties because of increasing evidence of its prominent role in field- and basin-scale transport. Experimental results have demonstrated the existence of heterogeneity of soil physical [Biggar and Nielsen, 1976; Bresler and Dagan, 1983; Sudicky, 1986; Freyberg, 1986; Goltz and Roberts, 1986; Hess et al., 1992] and soil chemical [Boekhold and van der Zee, 1992; Mackay et al., 1986a] parameters. Large solute transport experiments [Mackay et al., 1986b; Freyberg, 1986; LeBlanc et al., 1991] have shown the impact of spatially variable parameters, which emphasizes the need for understanding the effects of heterogeneity at local and regional scales [Dagan, 1986].

Considerable theoretical progress has been made with respect to flow and transport in physically heterogeneous porous media. Stochastic methods have been used to derive analytical expressions to describe flow and nonreactive solute transport in heterogeneous media by, e.g., Gutjahr and Gelhar [1981], Dagan [1984, 1988, 1989], Gelhar and

\footnotetext{
${ }^{1}$ Department of Soil Science and Plant Nutrition, Wageningen Agricultural University, Wageningen, Netherlands.

Dipartimento di Ingegneria Civile ed Ambientale, Università di Trento, Trent, Italy.

${ }^{3}$ Department of Civil Engineering, University of California, Berkeley.

"Istituto di Idraulica “G. Poleni," Università di Padova, Padua, Italy.
}

Copyright 1993 by the American Geophysical Union.

Raper number 93WR02305.

0043-1397/93/93WR-02305\$05.00
Axness [1983], Garabedian [1987], Neuman et al. [1987], Rubin [1990], and Rubin and Dagan [1992]. Use of a stochastic approach enables incorporation of uncertainty, resulting in expressions for first- and higher-order moments of the expected plume behavior. Various numerical studies have been performed to show the applicability of analytical approaches and to demonstrate the dispersive behavior of nonreactive transport in heterogeneous formations [e.g., Graham and McLaughlin, 1989; Rubin, 1990, 1991a, b; Tompson and Gelhar, 1990; Ababou et al., 1989; Bellin et al., 1992]. Numerical approaches based on stochastic theory often use uncoupled flow calculations and particle-tracking schemes to compute dispersion of a mass of solute in porous formations. This technique was used by Freeze [1975], Rubin [1990], Salandin and Rinaldo [1990], Valocchi [1989], and Bellin et al. [1992].

The above cited works primarily focused on heterogeneity of physical parameters, usually incorporated in a lumped variable, e.g., hydraulic conductivity. Less frequently, heterogeneity of soil chemical parameters has been taken into consideration. Examples where only spatial variability of chemical properties of the porous medium was taken into account are given by van der Zee [1990a], Chrysikopoulos et al. [1990, 1992], and Bosma and van der Zee [1993]. In the work by Chrysikopoulos et al. [1990, 1992], analytical expressions were derived for linearly adsorbing solute transport in one- and three-dimensional porous media with spatially variable retardation factor. Van der Zee [1990a] and Bosma and van der Zee [1993] considered nonlinearly adsorbing solute transport in chemically heterogeneous porous media. Van der Zee [1990a] used a semi-two-dimensional approach, assuming that heterogeneity in the horizontal plane dominates the heterogeneity in the direction of flow, to derive analytical expressions for the redistribution of a solute initially present in the topsoil. Bosma and van der Zee 
[1993] performed numerical Monte Carlo simulations to assess the effect of column scale heterogeneity on one- and semi-two-dimensional dispersive behavior. Both physical and chemical heterogeneity were taken into account by van der Zee and van Riemsdijk [1987], Dagan [1989], Russo [1989a, b], Cvetkovic and Shapiro [1990], Destouni and Cvetkovic [1991], Kabala and Sposito [1991], and Bellin et $a l$. [this issue]. The approaches used differ in the assumed correlation between physical and chemical properties, as well as in whether transport in multidimensional porous media was assumed, whether linear or nonlinear, equilibrium or nonequilibrium adsorption was assumed, and whether first-order decay was assumed. Also, results were obtained either analytically or numerically.

One- and semi-two-dimensional approaches were used by van der Zee and van Riemsdijk [1987], Russo [1989a, b], and Destouni and Cvetkovic [1991]. Russo [1989a, b] examined the effect of spatial variability of physicochemical interactions on transport of $\mathrm{Na} / \mathrm{Ca}$-chloride salts through a one-dimensional unsaturated soil. It was concluded that heterogeneity of soil properties in the unsaturated zone may enhance the heterogeneity of the field response. Van der Zee and van Riemsdijk [1987] derived analytical expressions for the penetration depth of a nonlinearly adsorbing solute, considering a semi-two-dimensional field with spatially variable water velocity, adsorption coefficient, and applied amount. The semi-two-dimensional field was modeled as an ensemble of noninteracting parallel stream tubes. Destouni and Cvetkovic [1991] showed double-peak behavior of the breakthrough of a kinetically linearly adsorbing solute in a semi-two-dimensional field with spatially variable water velocity and sorption rate coefficients.

Fully two- and three-dimensional analytical approaches were given by Dagan [1989], Cvetkovic and Shapiro [1990], Kabala and Sposito [1991], and Bellin et al. [this issue]. Dagan [1989] derived the asymptotic behavior of dispersion of linearly adsorbing solutes in heterogeneous media with spatially variable retardation factor perfectly correlated with spatially variable hydraulic conductivity. Cvetkovic and Shapiro [1990] considered nonequilibrium linear adsorption and examined perfectly correlated and uncorrelated sorption rate coefficients and hydraulic conductivity. They averaged the position of the solute in the transverse direction to derive an expression for the arrival time at a plane perpendicular to the direction of transport. Kabala and Sposito [1991] examined the behavior of a solute subject to spatially variable adsorption and first-order decay in a heterogeneous velocity field. They showed that the effective solute velocity differs from the ensemble average solute velocity. Bellin et al. [this issue] extended Dagan's [1989] theory for the dispersion of linearly adsorbing solutes in physically and chemically heterogeneous porous media. They derived expressions for the second-order moments of transport under ergodic conditions to assess the longitudinal and transverse spreading behavior as a function of time. Perfectly correlated and uncorrelated cases were considered.

This paper deals with numerical experiments to show the effect of spatially variable hydraulic conductivity in combination with a spatially variable sorption coefficient. Parameters that are used to describe the heterogeneities (i.e., mean sorption coefficient, correlation between physical and chemical properties, length of integral scales, variances of spatially variable parameters) are varied in the numerical calcu- lations. The numerical experiments are limited to a twodimensional domain due to computational restrictions. Additionally, the performance and applicability of the ana. lytical solutions derived in paper 1 [Bellin et al., this issue] are shown for the cases considered.

\section{Mathematical formulation of the Problem}

We consider transport of a reacting solute in a chemically and physically heterogeneous two-dimensional porous medium. The physical heterogeneity is represented by a spatially variable hydraulic conductivity whereas the chemical heterogeneity is due to a spatially variable adsorption coefficient. Although basically heterogeneity of these parameters is deterministic and caused by spatially variable physical and chemical properties, the complexity of heterogeneous structures and the problem of accounting for the variability and uncertainty call for a stochastic approach. Using such an approach, the spatially variable parameters are considered random space functions (RSF) with correlation [Dagan. 1989].

\subsection{Heterogeneous Flow}

To assess water flow through heterogeneous porous formations, several physical properties can be assumed spatially variable. According to experimental results [Freeze. 1975; Biggar and Nielsen, 1976; Warrick et al., 1977]. physical properties may be modeled by a lognormal distribution. Numerous studies have been performed with a lognormally distributed hydraulic conductivity [e.g., Dagan, 1984, 1988, 1989; Gelhar and Axness, 1983; Shapiro and Cvetkovic, 1988; Bellin et al., 1992] and a lognormally distributed scaling factor [e.g., Bresler and Dagan, 1979, 1983].

In view of generally accepted concepts we assume a lognormally spatially variable hydraulic conductivity $K(\mathbf{x})$, with $\mathrm{x}=\left(x_{1}, x_{2}\right)$. Similar to the procedure in paper 1 [Bellin et al., this issue] we assumed a normally distributed $\log$ conductivity $Y$, defined as $Y(x)=\ln [K(x)]$, with constant mean $\langle Y\rangle$ and variance $\sigma_{Y}^{2}$. To complete the necessary information of the stochastic variable $Y$, an isotropic exponential covariance function is assumed, defined as $C_{Y}(\mathbf{r})=$ $\left\langle Y^{\prime}(\mathbf{x}) Y^{\prime}(\mathbf{x}+\mathbf{r})\right\rangle$ with $Y^{\prime}(\mathbf{x})=Y(\mathbf{x})-\langle Y\rangle$ and given by [Black and Freyberg, 1987; Dagan, 1989; Bellin et al., 1992; Bosma and van der Zee, 1993; Bellin et al., this issue]

$$
C_{Y}(\mathbf{r})=\sigma_{Y}^{2} \exp \left(\frac{-|\mathbf{r}|}{l_{Y}}\right)
$$

where $r$ is the planar distance vector between two positions in the heterogeneous domain and $l_{Y}$ is the integral scale of the $\log$ conductivity. Equation (1) therefore describes the degree of correlation of two $Y$ values at a distance $|r|$.

Transport of reacting solute in heterogeneous porous media is governed by advection and diffusion. To describe advection of a moving solute, the water velocity at each point in the domain should be known. The random space function hydraulic conductivity causes the water velocity $\mathbf{v}(\mathbf{x})$ to be a RSF as well. To obtain a velocity field in a heterogeneous porous medium, Darcy's law in combination with the mass balance equation needs to be solved. The large difference in the characteristic times of flow and transport allows water flow to be considered in a steady state condi- 
tion. Following this hypothesis the velocity at position $\mathbf{x}$ can be obtained from Dagan [1989]:

$$
\begin{gathered}
\nabla \phi+\nabla Y \cdot \nabla \phi=\mathbf{J} \cdot \nabla Y \\
\Phi(\mathbf{x})=-\mathbf{J} \cdot \mathbf{x}+\phi(\mathbf{x}) \\
\mathbf{v}(\mathbf{x})=-\frac{K(\mathbf{x})}{\theta} \nabla \Phi(\mathbf{x})
\end{gathered}
$$

where $\Phi$ is the hydraulic head, $-J$ is the mean head gradient $J=(J, 0), \phi$ is the random head fluctuation with zero mean, and $\theta$ is the porosity. The statistical characterization of the velocity field viewed through (4) and (2) as a homogeneous random function is assumed complete with the knowledge of the first two moments, e.g., the mean $\left\langle v_{i}\right\rangle(i=1,2)$ and the covariance function $v_{i j}(\mathbf{r})=\left\langle\left(v_{i}(\mathbf{x})-\left\langle v_{i}\right)\right)\left(v_{j}(\mathbf{x}+\mathbf{r})-\right.\right.$ $\left.\left.\left\langle v_{j}\right\rangle\right)\right\rangle$, where $v_{i}(\mathbf{x})$ is the $i$ th component of the velocity at point x [Dagan, 1988; Rubin, 1990; Rubin and Dagan, 1992].

\subsection{Sorption Model Formulation}

Sorption at relatively low solute concentrations is usually dominated by surface complexation and/or electrostatic exchange. A recent review was given by Goldberg [1992]. At low levels, where the solute of interest hardly affects the equilibria for other compounds, the Freundlich equation, usually given as $C^{*}=K_{F} C^{n}$ (with $C$ and $C^{*}$ being the solute concentration in the liquid and in the solid phase, respectively, and $K_{F}$ and $n$ being Freundlich parameters), and the linear model, $C^{*}=K_{d} C$, where $K_{d}$ is the sorption coeffcient, are particularly useful. Since the sorption of heavy metals and pesticides is commonly weak to moderately nonlinear [Calvet et al., 1980; Chardon, 1984], a linearization of the Freundlich equation is favored for low concentrations. The cause for this is the non-Lipschitz behavior of $C^{*}=K_{F} C^{n}$ for $C=0$ which is physicochemically unrealistic. Recently, it was shown by Boekhold et al. [1993] that cadmium sorption by a sandy soil at low concentrations is practically linear. Although the assumption of linearity affects the transport behavior [van der Zee, 1990b; van Duijn and Knabner, 1992], it is not a point of interest in this study.

The parameter $K_{d}$ reflects all parameters that were not accounted for explicitly in the linear adsorption model, i.e., all but the concentration of the solute of interest. Because the adsorption maximum $C_{m}^{*}$ for permeable (e.g., sandy) media commonly depends on $f_{o m}$, the organic matter content, this is also the case for $K_{d}$. In fact, this was often shown to be the case for pesticides [Boesten, 1986]. Boekhold et al. [1993] showed for Cd that $K_{d}$ depended on $f_{o m}, p \mathrm{H}$, and other ionic solutes that either form complexes with $\mathrm{Cd}$ or compete for sorption sites on the matrix surface. It is plausible that these parameters affecting $K_{d}$ are random space functions [Beckett and Webster, 1971]. A demonstration was given by Boekhold et al. [1991] with regard to $f_{\text {om }}$ and $K_{d}$. They also showed that at larger concentrations (where $n<1$ ) the Freundlich equation gave good predictions of spatially variable sorption when the dependence of $K_{F}$ on $f_{o m}$ and $p \mathrm{H}$ was accounted for. The covariance functions of $f_{o m}$ and $p \mathrm{H}$ revealed different integral scales.

To model heterogeneity of the adsorption coefficient $K_{d}$, similar to the hydraulic conductivity $K$, a lognormal distribution is used. Because no a priori reasons exist to assume either negative or positive correlation between sorption parameters and the hydraulic conductivity [Destouni and Cvetkovic, 1991], several cases of correlation have been studied.

Regarding the cross correlation of $K_{d}$ and hydraulic conductivity, generally valid statements are not easily made. For different solutes, different factors (e.g., $p \mathrm{H}$, redox potential, ionic strength, matrix composition, cationic and anionic solution composition) may control the $K_{d}$ value [Boekhold et al., 1993; Goldberg, 1992; Bolt, 1982]. Whereas some factors may be positively correlated with hydraulic conductivity (such as clay content), negative or zero correlations are also feasible. Although in most practical situations the correlation between overall $K_{d}$ and hydraulic conductivity may appear to be most likely in the range of negative to mildly positive [Robin et al., 1991], there are no a priori reasons to exclude perfect (positive or negative) correlations.

If no correlation between $K_{d}$ and $K$ is assumed, heterogeneity of the adsorption coefficient can be described according to

$$
K_{d}(\mathbf{x})=K_{d}^{G} \exp [W(\mathbf{x})]
$$

where $K_{d}^{G}$ is the geometric mean of $K_{d}$ and $W$ is a normally distributed random variable with zero mean and variance $\sigma_{W}^{2}$. The autocovariance of $W, C_{W}$, is defined similar to (1):

$$
C_{W}(\mathbf{r})=\sigma_{W}^{2} \exp \left(\frac{-|\mathbf{r}|}{l_{W}}\right)
$$

with $l_{W}$ being the integral scale of $W$.

We adopted a general functional model to describe perfect correlation between hydraulic conductivity and adsorption, given by

$$
K_{d}(\mathbf{x})=K_{d}^{G}[\exp (Y(\mathbf{x}))]^{\beta}
$$

We account for various extreme cases that encompass likely correlations that may be revealed experimentally in the future. These are as follows: Case $A$ is perfect positive correlation $(\beta>0)$, and case $B$ is perfect negative correlation $(\beta<0)$. For $\beta=0$ we are dealing with the trivial case of homogeneous adsorption. With linear homogeneous adsorption, behavior is similar to the nonreactive case [Dagan, 1989; Bellin et al., 1992] except for a rescaling in time. If the cases $\beta=-1$ and $\beta=1$ are considered, (7) corresponds with the models used by van der Zee and van Riemsdijk [1987], Destouni and Cvetkovic [1991] and Bellin et al. [this issue].

Assuming a stationary velocity field and linear adsorption, transport of a reactive solute in a two-dimensional physically and chemically heterogeneous porous medium is described by the following equations:

$$
\begin{aligned}
& \frac{\partial C(\mathbf{x}, t)}{\partial t}+\frac{\partial C^{*}(\mathbf{x}, t)}{\partial t}=\nabla \cdot\left[\mathbf{D}_{\mathcal{U}} \cdot \nabla C(\mathbf{x}, t)\right] \\
&-\mathbf{v}(\mathbf{x}) \cdot \nabla C(\mathbf{x}, t) \\
& C^{*}(\mathbf{x}, t)=K_{d}(\mathbf{x}) \cdot C(\mathbf{x}, t)
\end{aligned}
$$

where $\mathbf{D}_{d}$ is the tensor of pore-scale dispersion and $\mathbf{v}(\mathbf{x})$ is the heterogeneous velocity field.

Transport in heterogeneous porous media can be represented in terms of spatial moments [Valocchi, 1989; Dagan, 1989]. Assuming the ergodic hypothesis to be valid, the first- 
and second-order spatial moments are equal to the statistical mean and variance of the particle trajectory, given by

$$
\begin{gathered}
\left\langle X_{j}(t)\right\rangle=\int_{-\infty}^{\infty} X_{j}(t) f(\mathbf{X}) d \mathbf{X} \\
X_{j l}(t)=\int_{-\infty}^{\infty}\left(X_{j}(t)-\left\langle X_{j}(t)\right\rangle\right)\left(X_{l}(t)-\left\langle X_{l}(t)\right\rangle\right) f(\mathbf{X}) d \mathbf{X}
\end{gathered}
$$

where $\mathbf{X}$ is the particle displacement and $f(\mathbf{X})$ is the probability density function of the trajectory $\mathbf{X}$.

Bellin et al. [this issue] extended Dagan's [1989] theory by deriving analytical solutions for the first and second longitudinal and transverse moments for the above correlated (for $\beta=-1$ and $\beta=1$ ) and uncorrelated cases. Their solutions were based on the linearized flow equation and on first-order approximations of adsorption. Therefore the solutions are only valid for small $\sigma_{Y}^{2}$ and $\sigma_{W}^{2}$. The assumptions correspond to those used by Dagan [1988, 1989] to derive first-order analytical solutions for the nonreactive case. For easy reference, the expressions derived in paper 1 [Bellin et al., this issue] for the second-order moments in the longitudinal $\left(X_{11}\right)$ and transverse $\left(X_{22}\right)$ directions are given here, i.e.,

$$
\begin{aligned}
X_{11}(\tau)= & 2 \frac{\left(K_{d}^{G}\right)^{2} l_{Y}^{2}}{\langle R\rangle^{2}} \exp \left(\sigma_{Y}^{2}\right)\left(\left[E i\left(\sigma_{Y}^{2}\right)-\gamma-\ln \left(\sigma_{Y}^{2}\right)\right] \tau\right. \\
& \left.+\sum_{m=1}^{\infty} \frac{\left(\sigma_{Y}^{2}\right)^{m}}{m^{2} m !} \exp (-m \tau)-\sum_{m=1}^{\infty} \frac{\left(\sigma_{Y}^{2}\right)^{m}}{m^{2} m !}\right) \\
& +\frac{4 K_{d}^{G} l_{Y}^{2}}{\langle R\rangle} \sigma_{Y}^{2} \exp \left(\sigma_{Y}^{2} / 2\right)[\tau-\ln (\tau)+E i(-\tau)-\gamma] \\
& +\sigma_{Y}^{2} l_{Y}^{2}\left[2 \tau-3 \ln (\tau)+\frac{3}{2}-3 \gamma\right. \\
& \left.+3\left(E i(-\tau)+\frac{\exp (-\tau)(1+\tau)-1}{\tau^{2}}\right)\right]
\end{aligned}
$$

for the correlated cases (minus sign for positive correlation and plus sign for negative correlation), and

$$
\begin{aligned}
& X_{11}(\tau)=2 \frac{\left(K_{d}^{G}\right)^{2}}{\langle R\rangle^{2}} \exp \left(\sigma_{W}^{2}\right) l_{W}^{2}\left(\frac { l _ { Y } } { l _ { W } } \left[E i\left(\sigma_{W}^{2}\right)-\gamma\right.\right. \\
& \left.\left.-\ln \left(\sigma_{W}^{2}\right)\right] \tau+\sum_{m=1}^{\infty} \frac{\left(\sigma_{W}^{2}\right)^{m}}{m^{2} m !} \exp \left(-m \frac{l_{Y}}{l_{W}} \tau\right)-\sum_{m=1}^{\infty} \frac{\left(\sigma_{W}^{2}\right)^{m}}{m^{2} m !}\right) \\
& +\sigma_{Y}^{2} l_{Y}^{2}\left[2 \tau-3 \ln (\tau)+\frac{3}{2}-3 \gamma\right. \\
& \left.+3\left(E i(-\tau)+\frac{\exp (-\tau)(1+\tau)-1}{\tau^{2}}\right)\right]
\end{aligned}
$$

for the uncorrelated cases. The expression for the transverse second-order moment is, for correlated and uncorrelated cases, given by

$$
\begin{aligned}
& X_{22}(\tau)=\sigma_{Y}^{2} l_{Y}^{2}[\ln (\tau)-\frac{3}{2}+\gamma-E i(-\tau) \\
&\left.+3\left(\frac{1}{\tau^{2}}-\frac{\exp (-\tau)(1+\tau)}{-\tau}\right)\right]
\end{aligned}
$$

In (12)-(14), $\tau$ is dimensionless time $\left(\tau=t V /\left.\langle R\rangle\right|_{Y}\right)$, $\gamma(\approx 0.577)$ is Euler's constant and $E \hat{i}(x)=\int_{-\infty}^{x} \exp (z) / z$ $d z$, for $x>0$ is the exponential integral. Observe that the transverse second-order moment (14) is influenced only by chemical heterogeneity through the mean retardation factor $\langle R\rangle$ in $\tau$.

\section{NUMERICAL EXPERIMENTS}

\subsection{Monte Carlo Approach}

Numerical simulations of transport in heterogeneous porous media may conveniently employ a Monte Carlo approach through which many independent realizations are generated, depending on the problem and the scale of heterogeneity. Bellin et al. [1992] demonstrated that as $\sigma_{Y}^{2}>$ 1 as many as 1500 realizations were necessary to stabilize the more sensitive transverse second moment. The approach utilized in this study is similar to the method used by Bellin et al. [1992].

For each case a series of Monte Carlo simulations was performed, using independent realizations of the same random fields. Four phases can be distinguished in each simulation: (1) generation of the random conductivity field, (2) solution of the flow equation to obtain the heterogeneous velocity field, (3) generation of the random adsorption field, and (4) calculation of solute transport.

The two-dimensional domain was discretized in triangular elements. Two adjacent elements contained the same value for the random variables. An efficient fast Fourier transform method [Gutjahr, 1989] was used to generate the random fields with prescribed correlation structure. The velocity field was obtained by solving the fully nonlinear flow equation (2)-(4) by a Galerkin finite element method [Bellin et al., 1992]. The generated random field of the adsorption coefficient and the calculated heterogeneous velocity field combine to the retarded velocity field, $\mathbf{v}^{R}(\mathrm{x})=\mathrm{v}(\mathrm{x}) / R(\mathrm{x})$, with $\boldsymbol{R}(\mathbf{x})$ being the spatially variable retardation coefficient given by $R(\mathrm{x})=1+K_{d}(\mathrm{x})$. It is worthwhile noting that approximations used to derive the analytical solutions [Bellin et al.. this issue] do not play a role in the numerical calculations.

Once the retarded velocity field is available, transport can be calculated by a particle-tracking scheme. A particle, which represents a certain amount of mass injected in the area, moves according to [Hockney and Eastwood, 1988; Tompson and Gelhar, 1990; Bellin, 1992]

$$
\mathbf{X}(t+\Delta t)=\mathbf{X}(t)+\mathbf{v}^{R}[\mathbf{X}(t)] \cdot \Delta t
$$

where $\mathbf{X}$ is the position of the particle. In (15) we neglected both molecular diffusion and pore-scale dispersion because the latter is assumed to be of less importance than the effect of the heterogeneous velocity field [Dagan, 1989; Bellin et al., 1992]. 
In each Monte Carlo realization the trajectory of only one single particle is recorded as a function of time. In contrast with numerical approaches in which many particles are tracked in one realization, the approach with one particle per realization guarantees independent trajectories of all particles. Using this approach, the ergodic requirement [Dagan, 1988,1989 ] is fully satisfied. The statistical moments (mean trajectory and longitudinal and transverse second moments) represent the spatial moments of a plume traveling in a heterogeneous domain and injected in a source of a size which is much larger in the transverse direction than the scale of heterogeneity [Dagan, 1990; Bellin et al., 1992].

The statistical moments of the trajectories are calculated numerically according to

$$
\begin{gathered}
\left\langle X_{j}(t)\right\rangle=\frac{1}{\mathrm{MC}} \sum_{i=1}^{\mathrm{MC}} X_{j}^{i}(t) \\
X_{j j}(t)=\frac{1}{\mathrm{MC}} \sum_{i=1}^{\mathrm{MC}}\left(X_{j}^{i}(t)-\left\langle X_{j}(t)\right\rangle\right)^{2}
\end{gathered}
$$

where $j$ denotes either the longitudinal ( $j=1$ ) or transverse $(j=2)$ direction and the number of Monte Carlo realizations is given by MC. The second-order moments $X_{j l}$, with $j \neq l$, have not been taken into account. An important aspect of this study deals with the ability of the analytical solutions for physical and chemical heterogeneous porous media (12)-(14) to describe $X_{11}$ and $X_{22}$ calculated with (16)-(17) for different heterogeneous cases.

\subsection{Simulation Cases}

Although our interest is the effect of physical and chemical heterogeneity on displacement behavior of a solute plume, some attention is given to the circumstances under which either physical or chemical heterogeneity dominates the transport process. Randomness of the field parameters hydraulic conductivity and sorption coefficient is considered. Spatially variable grain size distributions, organic matter content, $p \mathrm{H}$, and microscopic mechanisms determine the heterogeneity of both physical and chemical parameters. From a transport point of view, heterogeneity of the hydraulic conductivity and the sorption coefficient results in a spatially variable flow field with a spatially variable retardation factor. As was shown by a simple approximation [Dagan, 1989], the coefficient of variation of the longitudinal velocity $\left(\mathrm{CV}_{\tau}\right)$, which represents the basic spreading mechanism for the nonreactive case, depends only on $\sigma_{Y}^{2}$. The relative importance of physical and chemical heterogeneity can be assessed quantitatively by comparing the coefficients of variation (CV) of the velocity and the retardation factor. These parameters are considered representative for physical and chemical heterogeneity, respectively.

Bellin et al. [this issue] showed that for the reactive case the mean sorption coefficient plays an important role in the heterogeneity of the retarded velocity field. This is due to defining the retardation factor as $1+K_{d}$, which causes $K_{d}^{G}$ to remain in the expression for $C V_{R}$, given for the uncorrelated case by

$$
\mathrm{CV}_{R}=\frac{K_{d}^{G}\left[\exp \left(0.5 \sigma_{W}^{2}\right)\right]\left[\exp \left(\sigma_{W}^{2}\right)-1\right]^{0.5}}{1+K_{d}^{G} \exp \left(0.5 \sigma_{W}^{2}\right)}
$$

For the correlated cases, $\mathrm{CV}_{R}$ is given by (18) with $\sigma_{Y}^{2}$ instead of $\sigma_{W}^{2}$. Because the main spreading is the result of the random retarded velocity field, the variation coefficient of the velocity field should be compared with the variation coefficient of the retardation factor. Note that generally these variation coefficients are different from the variation coefficients of the field parameters $K$ and $K_{d}$.

The mean sorption coefficient plays an especially important role if the solute is not strongly sorbed, i.e., if $K G$ is small [Bellin et al., this issue]. Little sorption can be relevant in all kinds of environmental circumstances, depending on $p \mathrm{H}$, organic matter content, and background electrolyte concentration. Calculations have been performed with three values of $K_{d}^{G}$, namely, $0.2,1.26$, and 10.0 . These values were chosen to represent a small $\left(K_{d}^{G}=0.2\right)$ and a large ( $K_{d}^{G}=10.0$ ) mean sorption coefficient and to include the intermediate case for which $\mathrm{CV}_{R}=\mathrm{CV}_{v}$ if $\sigma_{Y}^{2}=\sigma_{W}^{2}=0.2$ $\left(K_{d}^{G}=1.26\right)$. The latter case describes the situation where physical and chemical heterogeneity are equal from a transport point of view. From the point of view of field parameters the condition for equal degrees of physical and chemical heterogeneity is met if the coefficients of variation of $K$ and $K_{d}$ are the same. This condition is fulfilled if $\sigma_{Y}^{2}=\sigma_{W}^{2}$; the value of $K_{d}^{G}$ is not relevant.

To show the effect of correlation and the performance of the analytical solutions (12)-(14), computations of correlated and uncorrelated cases have been performed. For the correlated cases, $\sigma_{Y}^{2}=0.2$ has been used. Equation (7) was used to determine the spatially variable sorption field. Positive correlation was simulated with $\beta=1$, whereas negative correlation was described with $\beta=-1$. Positively and negatively correlated calculations were performed for $K_{d}^{G}=$ $0.2, K_{d}^{G}=1.26$, and $K_{d}^{G}=10.0\left(\sigma_{Y}^{2}=0.2\right)$.

The computations of the uncorrelated cases were used to study the effect of $K_{d}^{G}, l_{Y} / l_{W}, \sigma_{W}^{2}$, and $\sigma_{Y}^{2}$. A reference case with $K_{d}^{G}=1.26, \sigma_{Y}^{2}=0.2, \sigma_{W}^{2}=0.2$, and $l_{Y}=l_{W}$ was defined so that $\mathrm{CV}_{R}=\mathrm{CV}_{v}$. The effect of the mean sorption coefficient was obtained by varying $K_{d}^{G}$ of the reference case $\left(K_{d}^{G}=0.2, K_{d}^{G}=10.0\right.$ ). The effect of the integral scale was examined with respect to the reference case with an equal degree of physical and chemical heterogeneity. Cases with $l_{Y}=0.5 l_{W}, l_{Y}=l_{W}$, and $l_{Y}=2 l_{W}$ were simulated. For these cases, $l_{Y}$ was kept constant and $l_{W}$ was varied. The effect of the degree of chemical heterogeneity was assessed for $\sigma_{W}^{2}=0.05,0.2,0.8$, and 1.6 . The remaining variables were similar to the reference case $\left(K_{d}^{G}=1.26, \sigma_{Y}^{2}=0.2\right.$, $\left.l_{Y}=l_{W}\right)$.

A large degree of physical heterogeneity was simulated with $\sigma_{Y}^{2}=1.6$ for all three $K_{d}^{G}$ values $(0.2,1.26,10.0)$. The remaining variables for these calculations were $\sigma_{W}^{2}=0.2$ and $l_{Y}=l_{W}$. A complete overview of the simulated cases is given in Table 1.

In addition to the above parameters, which determine heterogeneity of physical and chemical properties, other parameters were kept constant in all calculations. The size of the domain, expressed in integral scales, was $36 l_{Y}$ in the $x_{1}$ direction and $18 l_{y}$ in the $x_{2}$ direction. To minimize the effects of boundaries in the transport calculations, an inner core region was defined. The need for an inner core region, first detected by Rubin and Dagan [1989], is illustrated by Bellin et al. [1992], who showed the boundary effect on the velocity covariance. If a particle leaves the inner core, the realization is not taken into account. The size of the inner 
TABLE 1. Simulation Cases

\begin{tabular}{llllcc}
\hline Case & $K_{d}^{G}$ & $\sigma_{\tilde{Y}}^{2}$ & $\sigma_{W}^{2}$ & $l_{Y} / l_{W}$ & Correlation \\
\hline A1 & 0.2 & 0.2 & 0.2 & 1.0 & no \\
A2 & 0.2 & 0.2 & & 1.0 & + \\
A3 & 0.2 & 0.2 & & 1.0 & - \\
A4 & 0.2 & 1.6 & 0.2 & 1.0 & no \\
B1 & 1.26 & 0.2 & 0.2 & 1.0 & no \\
B2 & 1.26 & 0.2 & & 1.0 & + \\
B3 & 1.26 & 0.2 & & 1.0 & - \\
B4 & 1.26 & 0.2 & 0.2 & 0.5 & no \\
B5 & 1.26 & 0.2 & 0.2 & 2.0 & no \\
B6 & 1.26 & 0.2 & 0.05 & 1.0 & no \\
B7 & 1.26 & 0.2 & 0.8 & 1.0 & no \\
B8 & 1.26 & 0.2 & 1.6 & 1.0 & no \\
B9 & 1.26 & 1.6 & 0.2 & 1.0 & no \\
C1 & 10.0 & 0.2 & 0.2 & 1.0 & no \\
C2 & 10.0 & 0.2 & & 1.0 & + \\
C3 & 10.0 & 0.2 & & 1.0 & - \\
C4 & 10.0 & 1.6 & 0.2 & 1.0 & no \\
\hline
\end{tabular}

core $\left(29 l_{Y} \times 11 l_{Y}\right)$ was large enough to lead to only a few canceled realizations. The coordinates of the particle injection point are given by $x_{0}=\left(5 l_{Y}, 9 l_{Y}\right)$. A sketch of the domain used is shown in Figure 1.

In the calculations the time step was continuously modified in order to prevent the particle's crossing the cell boundaries within the time step. Similar to the calculations performed by Bellin et al. [1992], the boundary conditions used were no flux at $x_{2}=0$ and $x_{2}=18 l_{Y}$ and unit specific discharge in the $x_{1}$ direction at the nodes $\left(0, x_{2}\right)$ and $\left(36 l_{Y}\right.$, $\left.x_{2}\right)$. The condition $\phi=0$ is imposed for the node $x=(0,0)$. The dimensionless mean water velocity was 1 and 0 in the $x_{1}$ and $x_{2}$ directions, respectively.

\section{Results and Discussion}

Plume spreading of the cases given in Table 1 was assessed by calculation of the second-order moments, $X_{11}$ and $X_{22}$, as a function of time given by (17). For the sake of comparison, $X_{11}$ and $X_{22}$ are shown in dimensionless form by dividing $X_{11}$ and $X_{22}$ by $l_{Y}^{2}$. Time is made dimensionless by multiplying $t$ with $V /\langle R\rangle l_{Y}$, where $V$ is the mean Eulerian velocity and $\langle R\rangle$ is the mean retardation coefficient given by $1+K_{d}^{G} \exp \left(\sigma_{W}^{2} / 2\right)$. In the case of perfect correlation (positive or negative) $\langle R\rangle$ is given by $1+K_{d}^{G} \exp \left(\sigma_{Y}^{2} / 2\right)$.

In addition to the effects of variation of physical, chemi- cal, and statistical properties, the results are compared with the analytical solutions (12)-(14), for both correlated and uncorrelated cases. Similar to discussions given by Bellin et al. [1992] with respect to physical heterogeneity, comparison with an analytical solution gives insight on the effect of the assumptions made to derive the first-order approximations for the cases with physical and chemical heterogeneity.

In this paper we give full attention to plume spreading. The effect of physical and chemical heterogeneity on the mean position of the plume, denoted by its first-order moment, is not studied. The first-order approximation of the mean trajectory, expressed by $\left\langle X_{1}(t)\right\rangle=V t /\langle R\rangle$, predicts only an effect of $\langle R\rangle$. Numerical results for both nonreactive and reactive cases show small deviations from the analytical solution [Bellin, 1992; Bellin et al., 1992]. Larger deviations are observed for larger degrees of heterogeneities. However, these effects cannot be decomposed from effects that can be attributed to numerical inaccuracies [Bellin et al., 1992].

\subsection{Effect of the Mean Sorption Coefficient}

The effects of the mean sorption coefficient are illustrated in Figure 2. Values for $X_{11}$ and $X_{22}$ as a function of time are plotted for the cases A1, B1, and C1. Case A1 represents the case of little sorption and $\mathrm{C} 1$ of strongly adsorbed solutes, and for case B1, $K_{d}^{G}$ is chosen such that (with designated $\sigma_{\vec{Y}}^{2}$ and $\sigma_{Y}^{2}$ ) the variation coefficients of $R$ and $v$ are of the same order. Additionally, the numerical results from Bellin et al. [1992] which describe the nonreactive case (with physical heterogeneity only) are shown. The effect of chemical heterogeneity is apparent in Figure $2 a$. For $K_{d}^{G}=0.2$ a very small increase of plume spreading in the longitudinal direction can be seen with respect to the nonreactive case. The effects of chemical heterogeneity are in that case dominated by physical heterogeneity effects. Longitudinal plume spreading is enhanced by chemical heterogeneity for larger $K G$ values. The case with equal degrees of physical and chemical heterogeneity reveals that chemical heterogeneity cannot be ignored. Values for $X_{11}$ are in this case signifcantly larger than for the nonreactive case. For large $K_{d}^{G}$ values the chemical heterogeneity dominates the spreading process in the longitudinal direction. It is worthwhile mentioning that in view of Figure 2 of paper 1 [Bellin et al., this issue], a continued increase of $K_{d}^{G}$ will not increase $X_{11}$ due to insensitivity of $C_{R}$ for $K_{d}^{G}$ if $K_{d}^{G}>10.0$ (with unaltered

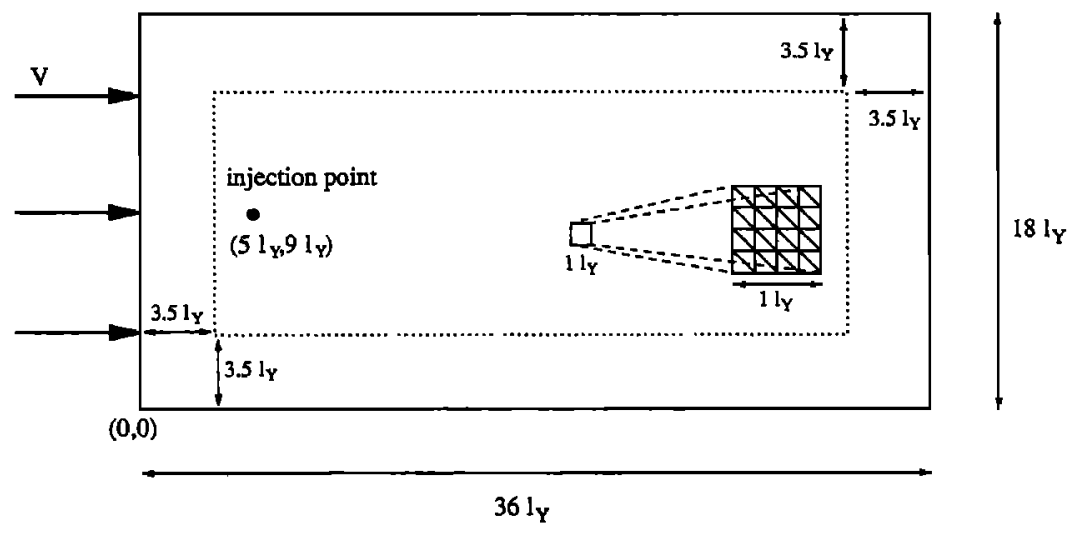

Fig. 1. Schematic representation of the two-dimensional transport domain. 

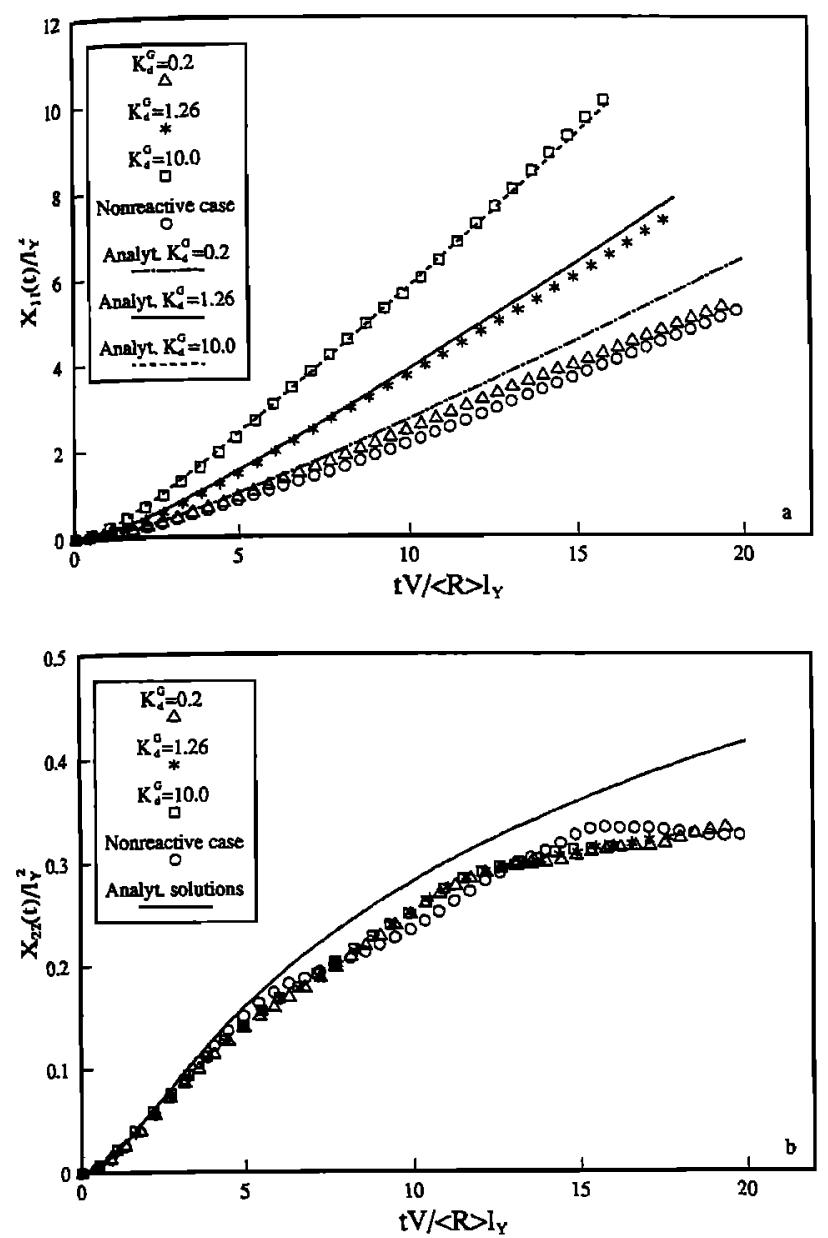

Fig. 2. Analytical (lines) and numerical (symbols) results of displacement in $(a)$ the longitudinal and $(b)$ transverse direction for various mean sorption coefficients (cases A1, B1, C1).

$\sigma_{W}^{2}$ ). Figure $2 b$ shows that the effects in the transverse direction are quite different. Corresponding to the analytical expression of the transverse second-order moment (14), $X_{22}$ is affected by chemical heterogeneity only by the scaling of time (division by $\langle R\rangle$ ). The numerical results of the nonreactive case and the cases $A 1, B 1$, and $C 1$ all follow the same pattern, which agrees with the analytical results of paper 1 [Bellin et al., this issue]. The deviating behavior of $X_{11}$ from the nonreactive case and the similar behavior of $X_{22}$ causes a different expected plume behavior for nonreactive and reactive solutes. Such different plume shapes were found in field experiments described by, among others, Mackay et al. [1986b].

Figure 2 also contains the results from the analytical solutions (13)-(14). The longitudinal moments, $X_{11}$, are well described by (13). First-order assumptions made in the derivations of (13)-(14) have a minor effect on $X_{11}$ for a relatively mild degree of heterogeneity $\left(\sigma_{W}^{2}=0.2\right)$. Interestingly, the agreement between analytical and numerical results improves as $K_{d}^{G}$ increases. Hence, Figure $2 a$ suggests a possibly compensative effect of the neglected higherorder terms in the linear expansion of $\mathbf{v}(\mathbf{x}) / R(\mathbf{x})$, used in the derivations of the analytical solutions (13)- (14), if $K_{d}^{G}$ values increase.

In Figure $2 b$, the curves of the analytical solutions of the cases shown are equal, which is in agreement with the theory. Despite some overestimation the analytical solutions [Dagan, 1989; Bellin et al., this issue] describe the numerical results reasonably well.

An alternative way to describe the numerical and analytical results is given in Figure 3. We demonstrate here the macroscopic longitudinal dispersion coefficient obtained by a numerical derivative of the calculated variances (i.e., $D_{11}$ $\left.=1 / 2 d X_{11} / d t\right)$. Despite some sensitivity for fluctuations, due to the calculation of the first derivative, the agreement between the analytical and numerical results is very good. The results show that for $K_{d}^{G}=0.2$ the limiting dispersion coefficient is slightly overestimated and that the agreement improves as $K_{d}^{G}$ increases.

\subsection{Effect of Correlation}

The results shown in Figures 2 and 3 were obtained for the uncorrelated case. To show the effect of perfect positive and negative correlation, results of cases $\mathrm{A} 1, \mathrm{~A} 2, \mathrm{~A} 3, \mathrm{B1}, \mathrm{B2}$, $\mathrm{B} 3, \mathrm{C1}, \mathrm{C} 2$, and $\mathrm{C} 3$ are presented in Figure 4 for various mean sorption coefficients. We see that in the longitudinal direction plume spreading is enhanced if $K$ and $K_{d}$ are negatively correlated, as is expected from the theory. Large conductivities combined with low sorption on one hand, and low conductivities combined with high sorption on the other, cause large solute spreading. This enhancement is stronger if the mean sorption coefficient $K_{d}^{G}$ is large. In the case of positive correlation between $K$ and $K_{d}$ the effects are somewhat different. As expected, for all cases, plume spreading in the longitudinal direction is smaller for the positively correlated case than for the uncorrelated case. However, the difference between the uncorrelated case and the positively correlated case is disproportionately different in the three cases considered $\left(K_{d}^{G}=0.2,1.26,10.0\right)$. In fact, in Figure 5 one can infer that in the case of positive correlation $X_{11}$ is larger for $K_{d}^{G}=0.2$ than for the cases with $K_{d}^{G}=1.26$ and $K_{d}^{G}=10.0$. Since we are dealing with two opposing effects, caused by physical and chemical heterogeneity, it is important to realize their magnitude. In the case with $K_{d}^{G}=0.2$, the effect of chemical heterogeneity is quite small, and therefore physical heterogeneity is not strongly affected by the opposing chemical heterogeneity. In

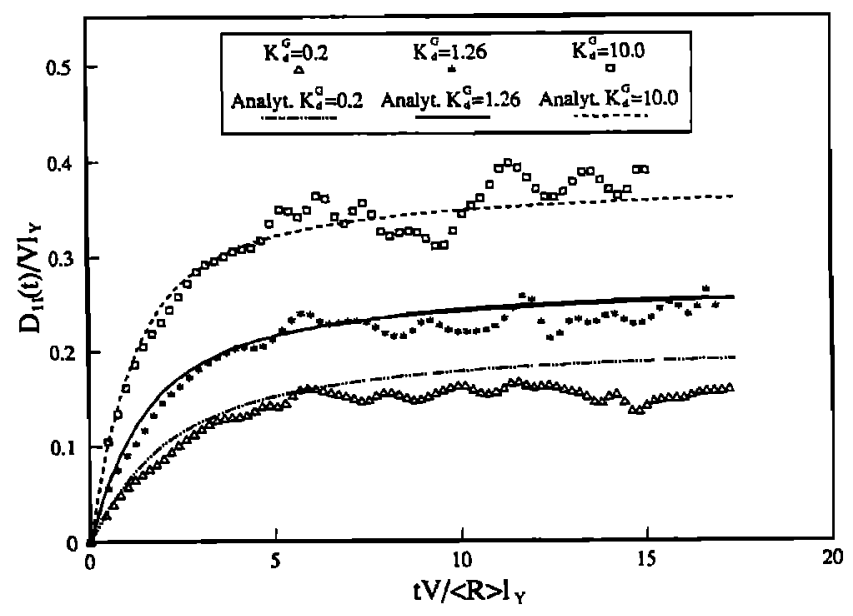

Fig. 3. Dispersion coefficients obtained by numerical differentiation for various mean sorption coefficients (cases A1, B1, C1) 

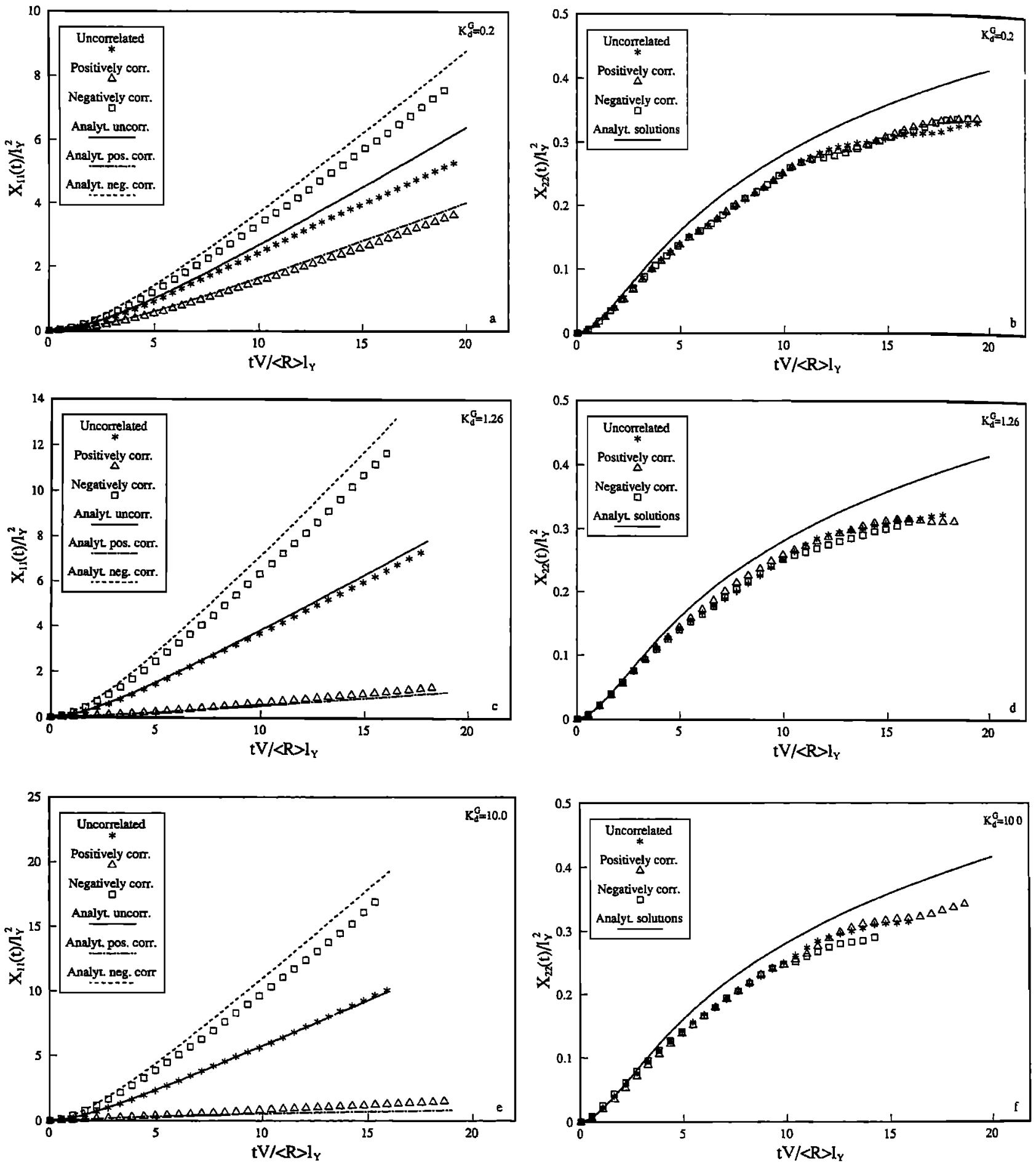

Fig. 4. Analytical (lines) and numerical (symbols) results of displacement in $(a, c, e)$ the longitudinal and $(b, d, f)$ transverse direction for positively correlated, negatively correlated and uncorrelated cases. In Figures $4 a$ and $4 b$, $K_{d}^{G}=0.2$ (cases A1, A2, A3); in Figures $4 c$ and $4 d, K_{d}^{G}=1.26$ (cases B1, B2, B3); in Figures $4 e, 4 f, K_{d}^{G}=10.0$ (cases $\mathrm{C} 1, \mathrm{C} 2, \mathrm{C} 3$ ).

the case of equal physical and chemical heterogeneity $\left(K_{d}^{G}\right.$ $=1.26)$, the effects are more balanced, and plume spreading in the longitudinal direction is relatively small. If chemical heterogeneity is larger than physical heterogeneity $\left(K_{d}^{G}=\right.$ $10.0), X_{11}$ is slightly larger than if $K_{d}^{G}=1.26$. Effects of physical heterogeneity are countered, but effects of the relatively large mean sorption coefficient remain, with the resulting increase in the longitudinal plume spreading. The chemical heterogeneity itself enhances longitudinal spreading if $K_{d}^{G}$ increases. Part of this enhancement is reduced by the positive correlation, causing large velocities at positions with strong adsorption and low velocities at positions with weak adsorption. Similar to the results of Figure 2, correlation (positive or negative) does not have an effect on spreading in the transverse direction.

The above results show that correlation between physical 

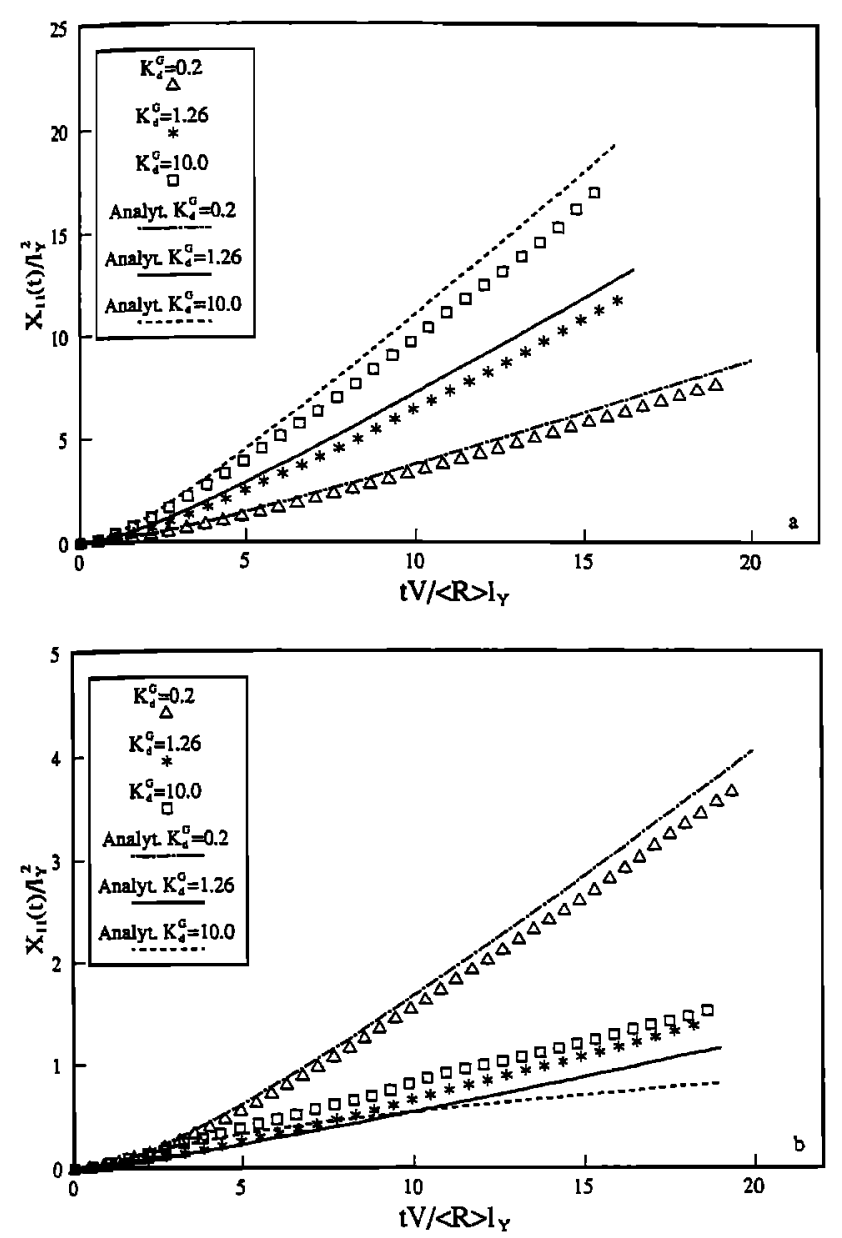

Fig. 5. Analytical (lines) and numerical (symbols) results of displacement in the longitudinal direction for $(a)$ negatively correlated and $(b)$ positively correlated cases for various mean sorption coefficients (Figure 5a: cases A3, B3, C3; Figure $5 b$ : cases A2, B2, C2).

and chemical parameters cannot be ignored. Although perfect correlation is unlikely to be found in the field, the results imply that moderate correlation may have a distinct effect as well. In Figures 4 and 5 the analytical solutions for the various cases are also shown. It can be seen that in the longitudinal direction, in general, the analytical results match the numerical results. Considering the negatively correlated cases, the analytical solution slightly overestimates the numerically calculated values. Although the performance of the analytical solution does not improve as $K_{d}^{G}$ increases, as was observed for the uncorrelated case (Figure 2), the deviations of $X_{11}$ for larger $K_{d}^{G}$ are relatively small. This may also be attributed to the compensative effect of the neglected higher-order terms in the derivation of the analytical solutions. Errors due to these effects may be enhanced by the negative correlation between $K$ and $K_{d}$. Higher $K_{d}^{G}$ values cause a slight increase of the overestimation of the analytical solution.

If $K$ and $K_{d}$ are positively correlated, the effects are opposite. An increase of $K_{d}^{G}$ causes, compared with the uncorrelated case, a decrease of the analytical solution with respect to the numerical results, resulting in an underestimation for $K_{d}^{G}>1.26$. Nevertheless, the deviations on an absolute scale are small (observe the scale in Figure $5 b$ ),
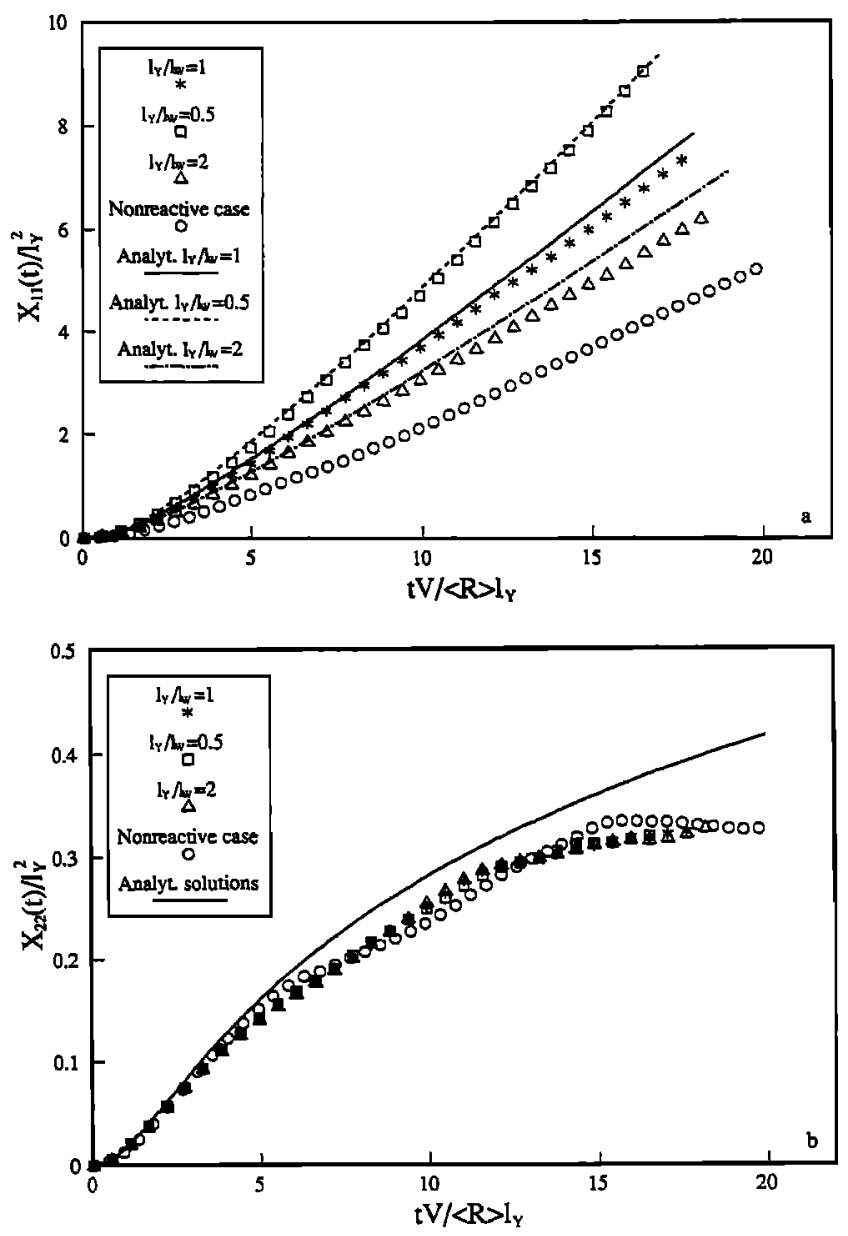

Fig. 6. Analytical (lines) and numerical (symbols) results of displacement in $(a)$ the longitudinal and $(b)$ transverse direction for various ratios of integral scales $\left(l_{Y} / l_{W}\right)$ (cases B1, B4, B5).

because positive correlation reduces the solute spreading. The better agreement of the analytical and numerical results for $K_{d}^{G}=0.2$ can be explained with the minor role of chemical heterogeneity if $K_{d}^{G}$ is small.

\subsection{Effect of Integral Scales}

The integral scale is an important parameter to describe the spatial structure of a spatially variable property. In combination with the mean and variance, it influences the effect of the heterogeneous variable. The integral scale is intuitively defined as a measure for the distance between two points beyond which the property is practically uncorrelated [Dagan, 1989].

The effect of the integral scale of the spatially variable chemical parameter can be revealed with cases B1, B4, and B5. The integral scale could not be multiplied by a factor larger than 2, due to limits of the domain size. Figure 6 shows numerical and analytical results of the cases B1, B4, and B5 and the numerical results of the nonreactive case [Bellin et al., 1992]. As expected, in Figure $6 a$ we show that a decrease of the chemical integral scale $\left(I_{W}\right)$ reduces spreading in the longitudinal direction. This reduction cannot continue beyond the lower limit represented by the nonreactive case (i.e., no chemical heterogeneity). On the other hand, an increase of $l_{W}$ enhances plume spreading in 
the longitudinal direction. A larger chemical integral scale generally increases the distance between large and small adsorption coefficients and therefore between quickly and more slowly moving particles. This results in larger longitudinal second-order moments. The results of the analytical solution show that for larger $l_{W}$ the numerical results are described well. For cases with lower chemical integral scales (i.e., $l_{Y} / l_{W}=1, l_{Y} / l_{W}=2$ ), the analytical solution somewhat overestimates the results of the numerical calculations.

The second-order moment in the transverse direction, $X_{22}$, is not sensitive to a change of the chemical integral scale (see Figure $6 b$ ). The transverse dispersion is determined by physical heterogeneity only, which was not altered in these cases. These results agree with derivations of Bellin et al. [this issue], who showed no functional relationship between $X_{22}$ and $l_{W}$.

\subsection{Effect of $\sigma_{Y}^{2}$ and $\sigma_{W}^{2}$}

The parameter which has been given the most attention in studies regarding transport in heterogeneous porous media is the variance of the spatial variable log conductivity, $\sigma_{Y}^{2}$ [Dagan, 1988, 1989; Bellin et al., 1992; Shapiro and Cvetkovic, 1988; Cvetkovic and Shapiro, 1989; Valocchi, 1989; Rubin, 1990; Selroos and Cvetkovic, 1992]. This parameter is used to describe the degree of physical heterogeneity. Due to the properties of the lognormal distribution, the variation coefficient of $Y$ is a function of $\sigma_{Y}^{2}$ only $\left(\mathrm{CV}_{Y}=\left[\exp \left(\sigma_{Y}^{2}\right)-\right.\right.$ 1] ${ }^{0.5}$ [van der Zee and Boesten, 1991]). Similarly, the parameter $\sigma_{W}^{2}$ determines the degree of chemical heterogeneity. Results from Figures $2-6$ show that chemical heterogeneity may have a large impact on spreading in the longitudinal direction. Therefore to demonstrate the effect of the degree of heterogeneity, several values of $\sigma_{W}^{2}$ and $\sigma_{Y}^{2}$ have been used. Cases B1, B6, B7, and B8 have been computed to demonstrate the effect of various degrees of chemical heterogeneity. Additionally, the effect of a large degree of physical heterogeneity $\left(\sigma_{Y}^{2}=1.6\right)$ is considered for three sorption levels (cases A4, B9, and C4).

Figure 7 reveals the longitudinal and transverse secondorder moment for four degrees of chemical heterogeneity, obtained by changing $\sigma_{W}^{2}$ with $K_{d}^{G}=1.26$. From the results in the longitudinal direction it can be seen that indeed different $\sigma_{W}^{2}$ values modify the spreading behavior. A strong increase of $X_{11}$ values is the result of a relatively mild increase of $\sigma_{W}^{2}$. For the case with $\sigma_{Y}^{2}=0.2$ (valid for all cases shown in Figure 7) and $\sigma_{W}^{2}=1.6$, chemical heterogeneity dominates the spreading process. On the other hand, Figure $7 b$ once again demonstrates the independence of transverse spreading from chemical heterogeneity. It can be seen that the numerical results in the transverse direction are not as smooth as the $X_{11}$ results. Bellin et al. [1992] already noticed slower convergence for the moments in the transverse direction in the nonreactive case.

The analytical results in Figure $7 a$ show that if $\sigma_{W}^{2}$ increases, larger deviations occur between the numerical results and the analytical solution. Errors due to first-order approximations are enhanced if the degree of heterogeneity increases. Apparently, if $K_{d}^{G}=1.26$ and $\sigma_{W}^{2}=1.6$, the higher-order effects are not strong enough to compensate for the overestimation of the analytical solution. Smaller deviations are observed in Figure 8 where results are shown for cases $\mathrm{A} 4, \mathrm{~B} 9$, and $\mathrm{C} 4$, with $\sigma_{\bar{Y}}^{2}=1.6, \sigma_{W}^{2}=0.2$ and $K_{d}^{G}=$
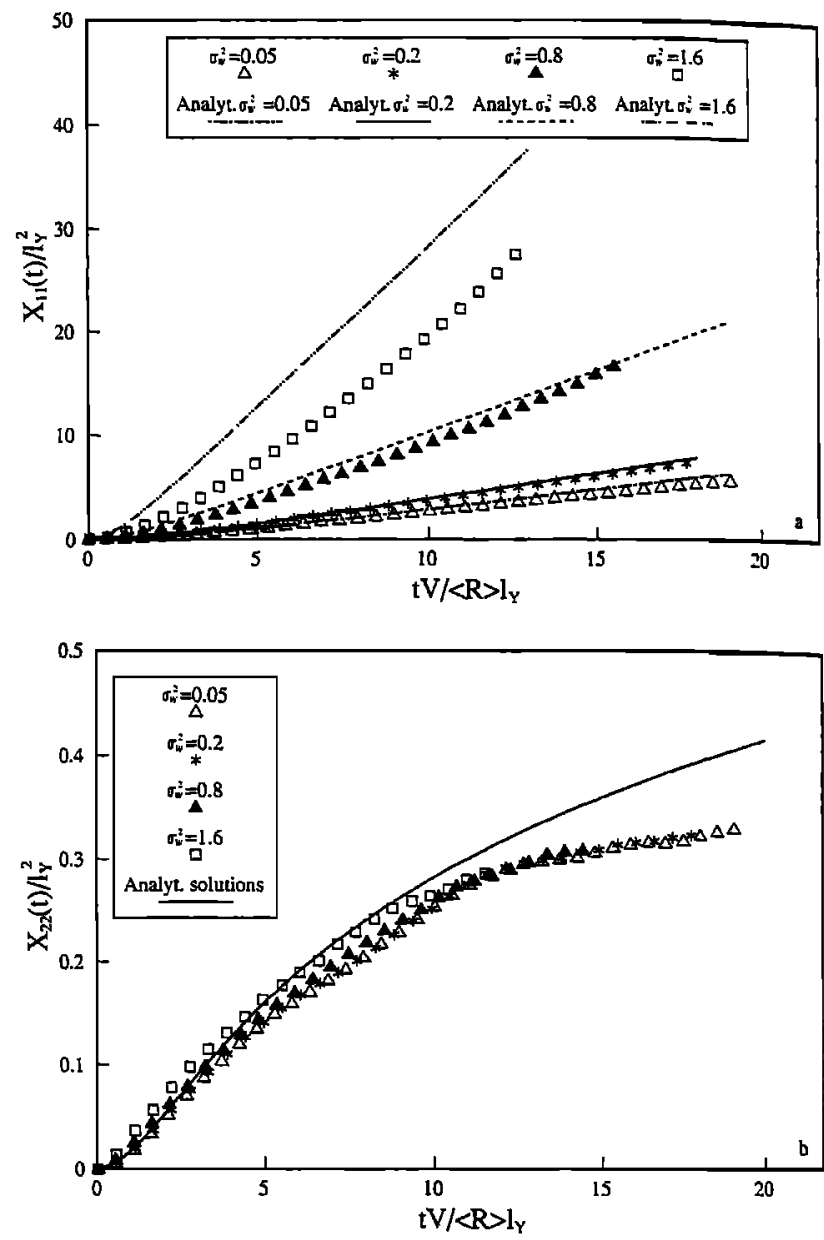

Fig. 7. Analytical (lines) and numerical (symbols) results of displacement in $(a)$ the longitudinal and $(b)$ transverse direction for various degrees of chemical heterogeneity $\left(\sigma_{W}^{2}\right)$ (cases B1, B6, B7. B8).

$0.2,1.26$, and 10.0. Figure $8 a$ illustrates that the analytical solution performs better for large $\sigma_{Y}^{2}$ than for large $\sigma_{W}^{2}$ values. With $\sigma_{Y}^{2}=1.6$, plume spreading in the longitudinal direction is more profound compared with cases where $\sigma_{\bar{Y}}^{2}=$ 0.2 (Figures 2-6). Interestingly, varying the $K_{d}^{G}$ values has only a minor impact on spreading in the longitudinal direction. Physical heterogeneity dominates, and only changes in $\sigma_{W}^{2}$ will enhance front spreading due to chemical heterogeneity. This agrees with results of Figure 2 in paper 1 [Bellin et al., this issue], which show that $\mathrm{CV}_{R} / \mathrm{CV}_{\imath}$ is hardly affected by $K_{d}^{G}$ if $\sigma_{Y}^{2}=1.6$ and $\sigma_{W}^{2}=0.2$. However, whether spreading in the longitudinal direction is dominated by either physical or chemical heterogeneity cannot be determined only by taking into account the chosen values for $\sigma_{Y}^{2}$ and $\sigma_{W}^{2}$. Previous sections have illustrated that the impact of correlation and of integral scales cannot be ignored.

Figure $8 b$ shows that an increase of $\sigma_{Y}^{2}$ influences the amount of spreading in the transverse direction. The $X_{21}$ values are significantly higher compared with cases computed with $\sigma_{Y}^{2}=0.2$ (e.g., Figure $2 b$ ). Again, changing $K_{d}^{G}$ has no effect on $X_{22}$. Results from the analytical solution demonstrate that an increasing degree of physical heterogeneity hardly affects the applicability of the solution. The 

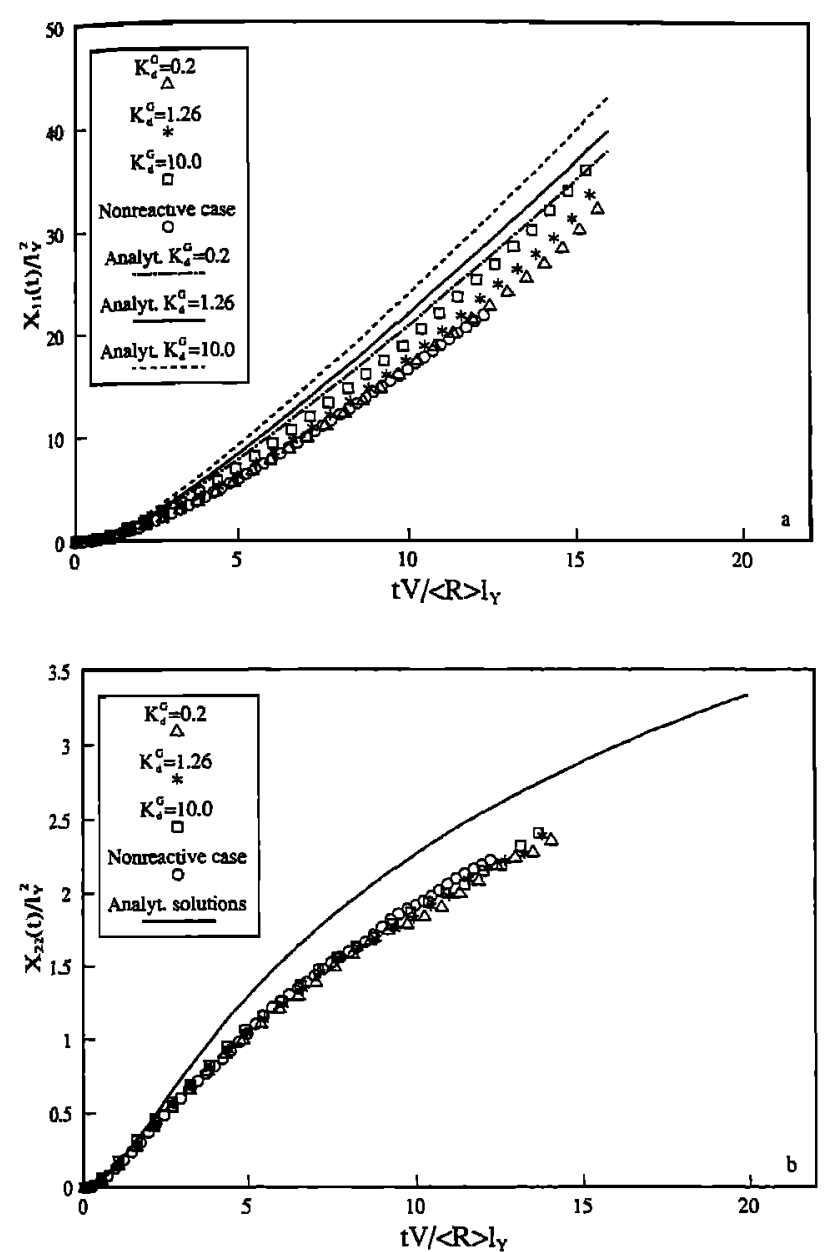

Fig. 8. Analytical (lines) and numerical (symbols) results of displacement in $(a)$ the longitudinal and $(b)$ transverse direction with large physical heterogeneity $\left(\sigma_{Y}^{2}=1.6\right)$ for various mean sorption coefficients (cases A4, B9, C4).

deviations found in all figures between the analytical solutions derived by Bellin et al. [this issue] and the actual numerical results are not significantly larger than the corresponding deviations for the nonreactive case [Rubin, 1990; Bellin et al., 1992]. This justifies the use of the analytical solution for correlated and uncorrelated cases with physical and chemical heterogeneity in the parameter range considered herein.

\section{CONClusions}

Numerical simulations were performed to assess the effect of physical and chemical heterogeneity on reactive solute transport. Physical heterogeneity was modeled by assuming a random variation with spatial correlation of the hydraulic $\log$ conductivity. Chemical heterogeneity was described in a similar manner assuming a random variation of the natural logarithm of the sorption coefficient. The results of the numerical Monte Carlo calculations were compared with analytical solutions for uncorrelated and for correlated cases (correlation between the hydraulic conductivity and the adsorption coefficient). Several values of the sorption coefficient, different ratios of chemical and physical integral scales, and several values for $\sigma_{Y}^{2}$ and $\sigma_{W}^{2}$ were considered. Solute spreading was described in terms of the second-order moments in the longitudinal and the transverse direction. We conclude from the results that stronger sorption (larger $K_{d}^{G}$ ) enhances the spreading in the longitudinal direction, for cases with both uncorrelated and correlated sorption coefficient and hydraulic conductivity. In the case of positively correlated $K_{d}$ and $K$, longitudinal spreading is reduced with respect to the uncorrelated and negatively correlated case. The parameters $K_{d}^{G}, \sigma_{W}^{2}, l_{W}, \sigma_{Y}^{2}$, and $l_{Y}$ determine the contribution of chemical and physical heterogeneity. If both contributions are of equal magnitude, a minimal degree of solute spreading is observed. Increasing the chemical integral scale enhances longitudinal solute spreading due to a larger distance between quickly and slowly moving particles. As expected, enhanced longitudinal spreading is also observed with larger coefficients of variation of either the hydraulic conductivity or the sorption coefficient (by increasing $\sigma_{Y}^{2}$ and $\sigma_{W}^{2}$ ). In agreement with analytical findings in paper 1 [Bellin et al., this issue], chemical heterogeneity only influences the time scale of the transverse solute spreading. Differences in correlation, $K_{d}^{G}, \sigma_{W}^{2}$, and integral scale do not increase or decrease values of the transverse second-order moment. Moreover, we conclude that the analytical solutions can describe solute displacement in physically and chemically heterogeneous porous media for a wide range of parameter values. An increase of the mean sorption coefficient generally improves the performance of the analytical solution, due to a compensative effect of the neglected higher-order terms as $K_{d}^{G}$ increases. If $\sigma_{Y}^{2}$ and $\sigma_{W}^{2}$ increase, the analytical solution overestimates the numerically obtained results. However, with $\sigma_{W}^{2}$ up to 0.8 and $\sigma_{Y}^{2}$ up to 1.6 , the analytical solution still agrees well with the numerical calculations. Despite some overestimation the numerical results of the transverse second-order moment are all well described by the analytical solution.

Acknowledgments. This work was partly funded by Directorate General Science, Research and Development of the Commission of the European Communities, via the European Community Environmental Research Program STEP-CT900031. The Italian group also acknowledges support from CNR-GNDCI (Linea 5).

\section{REFERENCES}

Ababou, R., D. McLaughlin, L. W. Gelhar, and A. F. B. Tompson, Numerical simulation of three-dimensional saturated flow in randomly heterogeneous porous media, Transp. Porous Media, 4, 549-565, 1989.

Beckett, P. H. T., and R. Webster, Soil variability: A review, Soils Fert., 34, 1-15, 1971.

Bellin, A., Un contributo allo studio del trasporto di soluti passivi e reattivi in formazioni porose eterogenee, Ph.D. thesis, Univ. di Trento, Trent, Italy, 1992.

Bellin, A., P. Salandin, and A. Rinaldo, Simulation of dispersion in heterogeneous porous formations: Statistics, first-order theories, convergence of computations, Water Resour. Res., 28, 22112227, 1992.

Bellin, A.. A. Rinaldo, W. J. P. Bosma, S. E. A. T. M. van der Zee, and $Y$. Rubin, Linear equilibrium adsorbing solute transport in physically and chemically heterogeneous porous formations, 1 , Analytical solutions, Water Resour. Res., this issue.

Biggar, J. W., and D. R. Nielsen, Spatial variability of the leaching characteristics of a field soil, Water Resour. Res., 12, 78-84, 1976.

Black, T. C., and D. L. Freyberg, Stochastic modeling of vertically averaged concentration uncertainty in a perfectly stratified aquifer, Water Resour. Res., 23, 997-1004, 1987.

Boekhold, A. E., and S. E. A. T. M. van der Zee, Significance of soil chemical heterogeneity for spatial behaviour of cadmium in field soils, Soil Sci. Soc. Am. J., 56, 747-754, 1992. 
Boekhold, A. E., S. E. A. T. M. van der Zee, and F. A. M. de Haan, Spatial patterns of cadmium contents related to soil heterogeneity, Water, Air, Soil Pollut., 57-58, 479-488, 1991.

Boekhold, A. E., E. J. M. Temminghoff, and S. E. A. T. M. van der Zee, Influence of electrolyte composition and $p \mathrm{H}$ on cadmium sorption by an acid sandy soil, J. Soil Sci., 44, 85-96, 1993.

Boesten, J. J. T. I., Behaviour of herbicides in soil: Simulation and experimental assessment, Ph.D. thesis, 263 pp., Wageningen Agric. Univ, Wageningen, Netherlands, 1986.

Bolt, G. H., Movement of solutes in soil: Principles of adsorption/ exchange chromatography, in Soil Chemistry, vol. B., PhysicoChemical Models, edited by G. H. Bolt, pp. 285-348, Elsevier Science, New York, 1982.

Bosma, W. J. P., and S. E. A. T. M. van der Zee, Transport of reacting solute in a one-dimensional chemically heterogeneous porous medium, Water Resour. Res., 29, 117-131, 1993.

Bresler, E., and G. Dagan, Solute dispersion in unsaturated soil at field scale, II, Application, Soil Sci. Soc. Am. J., 43, 467-472, 1979.

Bresler, E., and G. Dagan, Unsaturated flow in spatially variable fields, 2, Application of water flow models to various fields, Water Resour. Res., 19, 429-435, 1983.

Calvet, R., M. Tercé, and J. C. Arvieu, Adsorption des pesticides par les sols et leurs constituants, III, Caractéristiques générals de l'adsorption des pesticides, Ann. Agron., 3I, 239-257, 1980.

Chardon, W. J., Mobiliteit van cadmium in de bodem, Ph.D. thesis, 200 pp., Wageningen Agric. Univ., Wageningen, Netherlands, 1984.

Chrysikopoulos, C. V., P. K. Kitanidis, and P. V. Roberts, Analysis of one-dimensional solute transport through porous media with spatially variable retardation factor, Water Resour. Res., 26, 437-446, 1990.

Chrysikopoulos, C. V., P. K. Kitanidis, and P. V. Roberts, Generalized Taylor-Aris moment analysis of the transport of sorbing solutes through porous media with spatially-periodic retardation factor, Transp. Porous Media, 7, 163-185, 1992.

Cvetkovic, V. D., and A. M. Shapiro, Solute advection in stratified formations, Water Resour. Res., 25, 1283-1289, 1989.

Cvetkovic, V. D., and A. M. Shapiro, Mass arrival of sorptive solute in heterogeneous porous media, Water Resour. Res., 26, 2057-2067, 1990.

Dagan, G., Solute transport in heterogeneous porous formations, $J$. Fluid Mech., 145, 151-177, 1984.

Dagan, G., Statistical theory of groundwater flow and transport: Pore to laboratory, laboratory to formation, and formation to regional scale, Water Resour. Res., 22, 120S-134S, 1986.

Dagan, G., Time-dependent macrodispersion for solute transport in anisotropic heterogeneous aquifers, Water Resour. Res., 24, 1491-1500, 1988.

Dagan, G., Flow and Transport in Porous Formations, SpringerVerlag, New York, 1989.

Dagan, G., Transport in heterogeneous formations: Spatial moments, ergodicity, and effective dispersion, Water Resour. Res., 26, 1281-1290, 1990.

Destouni, G., and V. Cvetkovic, Field scale mass arrival of sorptive solute into the groundwater, Water Resour. Res., 27, 1315-1325, 1991.

Freeze, R. A., A stochastic-conceptual analysis of one-dimensional groundwater flow in nonuniform homogeneous media, Water Resour. Res., 11, 725-741, 1975.

Freyberg, D. L., A natural gradient experiment on solute transport in a sand aquifer, 2, Spatial moments and the advection and dispersion of nonreactive tracers, Water Resour. Res., 22, 2031$2046,1986$.

Garabedian, S. P., Large-scale dispersive transport in aquifers: Field experiments and reactive transport theory, Ph.D. thesis, Mass. Inst. of Technol., Cambridge, 1987.

Gelhar, L. W., and C. L. Axness, Three-dimensional stochastic analysis of macrodispersion in aquifers, Water Resour. Res., 19, $161-180,1983$.

Goldberg, S., Use of surface complexation models in soil chemical systems, Adv. Agron., 47, 234-329, 1992.

Goltz, M. N., and P. V. Roberts, Interpreting organic transport data from a field experiment using physical nonequilibrium models, $J$. Contam. Hydrol., 1, 77-93, 1986.

Graham, W., and D. McLaughlin, Stochastic analysis of nonstation- ary subsurface solute transport, 1, Unconditional moments, Wa ter Resour. Res., 25, 215-232, 1989.

Gutjahr, A. L., Fast Fourier transforms for random field generation, project report, contract 4-RS of Los Alamos National Laboratory, N. M. Inst. of Min. and Technol., Socorro, 1989.

Gutjahr, A. L., and L. W. Gelhar, Stochastic models of subsurface flow: Infinite versus finite domains and stationarity, Water Re. sour. Res., 17, 337-350, 1981.

Hess, K. M., S. H. Wolf, and M. A. Celia, Large-scale natural gradient tracer test in sand and gravel, Cape Cod, Massachusetts, 3 , Hydraulic conductivity variability and calculated macrodispersivities, Water Resour. Res., 28, 2011-2027, 1992.

Hockney, R. W., and J. W. Eastwood, Computer Simulation Using Particles, Adam Hilger, Bristol, England, 1988.

Kabala, Z. J., and G. Sposito, A stochastic model of reactive solute transport with time-varying velocity in a heterogeneous aquifer. Water Resour. Res., 27, 341-350, 1991.

LeBlanc, D. R., S. P. Garabedian, K. M. Hess, L. W. Gelhar, R. D. Quadri, K. G. Stollenwerk, and W. W. Wood, Large-scale natural gradient tracer test in sand and gravel, Cape Cod, Massachusetts, 1, Experimental design and observed tracer movement, Water Resour. Res., 27, 895-910, 1991.

Mackay, D. M., W. P. Ball, and M. G. Durant, Variability of aquifer sorption properties in a field experiment on groundwater transport of organic solutes: Methods and preliminary results, J. Contam. Hydrol., I, 119-132, 1986 e.

Mackay, D. M., D. L. Freyberg, P. V. Roberts, and J. A. Cherry, A natural gradient experiment on solute transport in a sand aquifer, 1, Approach and overview of plume movement, Water Resour. Res., 22, 2017-2029, 1986b.

Neuman, S. P., C. L. Winter, and C. M. Newman, Stochastic theory of field-scale Fickian dispersion in anisotropic porous media, Water Resour. Res., 23, 453-466, 1987.

Robin, M. J. L., E. A. Sudicky, R. W. Gillham, and R. G. Kachanoski, Spatial variability of strontium distribution coefficients and their correlation with hydraulic conductivity in the Canadian Forces Base Borden aquifer, Water Resour. Res., 27, 2619-2632, 1991.

Rubin, Y., Stochastic modeling of macrodispersion in heterogeneous porous media, Water Resour. Res., 26, 133-141, 1990.

Rubin, Y., Prediction of tracer plume migration in disordered porous media by the method of conditional probabilities, Water Resour. Res., 27, 1291-1308, $1991 a$.

Rubin, Y., Transport in heterogeneous porous media: Prediction and uncertainty, Water Resour. Res., 27, 1723-1738, $1991 b$.

Rubin, Y., and G. Dagan, Stochastic analysis of boundary effects on head spatial variability in heterogeneous aquifers, 2 , Impervious boundary, Water Resour. Res., 25, 707-712, 1989.

Rubin, Y., and G. Dagan, A note on head and velocity covariances in three-dimensional flow through heterogeneous anisotropic porous media, Water Resour. Res., 28, 1463-1470, 1992.

Russo, D., Field-scale transport of interacting solutes through the unsaturated zone, 1, Analysis of the spatial variability of the transport properties, Water Resour. Res., 25, 2475-2485, 1989 a.

Russo, D., Field-scale transport of interacting solutes through the unsaturated zone, 2, Analysis of the spatial variability of the field response, Water Resour. Res., 25, 2487-2495, 1989 b.

Salandin, P., and A. Rinaldo, Numerical experiments on dispersion in heterogeneous porous media, in Computational Methods in Subsurface Hydrology, edited by G. Gambolati et al., pp. 495501, Springer-Verlag, New York, 1990.

Selroos, J. O., and V. Cvetkovic, Modeling solute advection coupled with sorption kinetics in heterogeneous formations, Water Resour. Res., 28, 1271-1278, 1992.

Shapiro, A. M., and V. D. Cvetkovic, Stochastic analysis of solute arrival time in heterogeneous porous media, Water Resour. Res.. 24, 1711-1718, 1988.

Sudicky, E. A., A natural gradient experiment on solute transport in a sand aquifer: Spatial variability of hydraulic conductivity and its role in the dispersion process, Water Resour. Res., 22, 2069-2082, 1986.

Tompson, A. F. B., and L. W. Gelhar, Numerical simulation of solute transport in three-dimensional, randomly heterogeneous porous media, Water Resour. Res., 26, 2541-2562, 1990.

Valocchi, A. J., Spatial moment analysis of the transport of kinets- 
cally adsorbing solutes through stratified aquifers, Water Resour. Res., 25, 273-279, 1989.

van $\operatorname{der}$ Zee, S. E. A. T. M., Analysis of solute redistribution in a heterogeneous field, Water Resour. Res., 26, 273-278, 1990a.

van der Zee, S. E. A. T. M., Analytical traveling wave solutions for transport with nonlinear and nonequilibrium adsorption, Water Resour. Res., 26, 2563-2578, 1990b. (Correction, Water Resour. Res., 27, 983, 1991.)

van der Zee, S. E. A. T. M., and J. J. T. I. Boesten, Effects of soil heterogeneity on pesticide leaching to groundwater, Water $R e$ sour. Res., 27, 3051-3063, 1991.

van der Zee, S. E. A. T. M., and W. H. van Riemsdijk, Transport of reactive solute in spatially variable soil systems, Water Resour. Res., 23, 2059-2069, 1987.

van Duijn, C. J., and P. Knabner, Travelling waves in the transport of reactive solutes through porous media: Adsorption and binary exchange, 2, Transp. Porous Media, 8, 199-226, 1992.
Warrick, A. W., G. J. Mullen, and D. R. Nielsen, Scaling fieldmeasured soil hydraulic properties using a similar media concept, Water Resour. Res., 13, 355-362, 1977.

A. Bellin, Dipartimento di Ingegneria Civile ed Ambientale, Università di Trento, Mesiano di Povo, I-38050 Trento, Italy.

W. J. P. Bosma and S. E. A. T. M. van der Zee, Department of Soil Science and Plant Nutrition, Wageningen Agricultural University, P. O. Box 8005, 6700 EC Wageningen, Netherlands.

A. Rinaldo, Istituto di Idraulica "G. Poleni," Università di Padova, via Loredan, 20, I-35131 Padova, Italy.

\section{(Received December 22, 1992 revised June 18, 1993;} accepted August 10, 1993.) 